POS $\quad$ PROCEEDINGS

\title{
Brittle - Ductile Transition and Scatter in Fracture Toughness of Ferritic Steels
}

\section{S. J. Noronha*}

Mechanical \& Aerospace Engineering Department, University of California, Los Angeles 420 Westwood Plaza, Los Angeles, California, USA

E-mail: snoronha@ucla.edu

\section{N. M. Ghoniem}

Mechanical \& Aerospace Engineering Department, University of California, Los Angeles 420 Westwood Plaza, Los Angeles, California, USA

E-mail: ghoniem@ucla.edu

Here we analyze a dislocation simulation model for the brittle-to-ductile transition of Ferritic Steels, a typical multiphase material. The crack tip plastic zones are simulated as arrays of discrete dislocations emitted from crack-tip sources and equilibrated against the friction stress due to lattice and obstacles. The crack-tip gets blunted and the emitted dislocation arrays modify the elastic field of the crack. The combined stress field of the crack and the emitted dislocations describe an elasto-plastic crack field. The simulated crack system involves microcracks embedded in the plastic zone of a macrocrack. The inherent scatter in fracture toughness measurements are studied by using a size distribution for microcracks, distributed on the plane of the macrocrack. The scatter in fracture toughness measurements is found to be an effect of the size distribution of microcracks rather than their spatial distribution on the matrix ahead of the crack plane.

International Conference on Statistical Mechanics

of Plasticity and Related Instabilities

Indian Institute of Science, Bangalore

August 29-September 2, 2005

\footnotetext{
${ }^{*}$ Speaker
} 


\section{Introduction}

Predicting the brittle-ductile transition in multiphase materials like steels pose a scientific and technological challenge. Factors that influence the transition include change in fundamental material properties of the Fe matrix with temperature as well as the microstructural properties like grain sizes, carbide size distribution, etc. Typical microstructure of these materials have brittle precipitates (like carbides and sulphides) embeded in BCC Fe matrix. Now it is a well known fact that fracture in these materials at low temperature is originated in microcracks; and propragtion of these microcracks to the matrix is the critical step that lead to fracture [1].

Discrete dislocation simulations of crack-tip plasticity were successful in predicting the brittle-ductile transition (BDT) of simple single crystalline materials e.g.[2],[3]. The advantage of this approach over continuum methods is that the fundamental material properties such as dislocation velocity and their interactions can be treated dynamically. It has been found that the dislocation mobility plays a significant role in determining the transition temperature [3]. However, the variation of dislocation mobility alone cannot explain the BDT behavior of microstructurally complex materials like steels [4]. Recently we introduced a discrete dislocation simulation model in which the effects of blunting are incorporated by using elastic stress fields of blunted cracks which captures the increase in fracture toughness around the transition temperature [5]. Here we analyse the model to study the observed scatter in the measured fracture toughness values around the ductile-brtittle transition temperature (DBTT).

From numerous careful experimental studies conducted on the BDT behavior of steels it is now established that: (a) the measured microscopic fracture stress (at microcracks) is found to be several orders of magnitude higher than that of pure Griffith value [7],[8]; (b) several cracked brittle particles are found to be present in the broken samples [7],[9]; (c) pre-cracks (macrocracks) blunt substantially before the fracture of the specimen occurs at the transition region [10]; and (d) the size of carbides present in the matrix has a Poisson type distribution [11]. These observations were considered and accounted for in the simulations presented here.

\section{Model \& Method of Calculation}

Based on the above experimental observations, the present model has the following features:

(1) The emission of dislocations and subsequent shielding at the microcrack tip,

(2) A distribution of mircocracks on the crack plane of a macrocrack, ahead of it. 
(3) Cleavage propagation from any one of these microcracks near the main crack leads to failure,

(4) Blunting of macrocrack is accounted for by modifying the elastic crack-tip field of sharp crack, and

(5) The size distribution of microcracks is assumed to follow Posisson distribution. As shown schematically in Figure 1a, the macrocrack is assumed to be semi-infinite, with dislocation sources close to the crack-tip. Dislocations are emitted simultaneously along the two slip planes, symmetrically oriented to the crack plane and inclined at $70.5^{\circ}$ to the crack plane. The initial source position $\left(x_{0}\right)$ along the slip plane is chosen to be $4 b$, where $b$ is the magnitude of Burgers vector. As the load is increased dislocations are emitted and the crack-tip gets blunted, which is schematically illustrated in figure 1b. The radius of the blunted crack $\rho$ is taken to be equal to $N b \sin \alpha$, where $N$ is the number of dislocations and $\alpha$ is the slip plane angle. As blunting

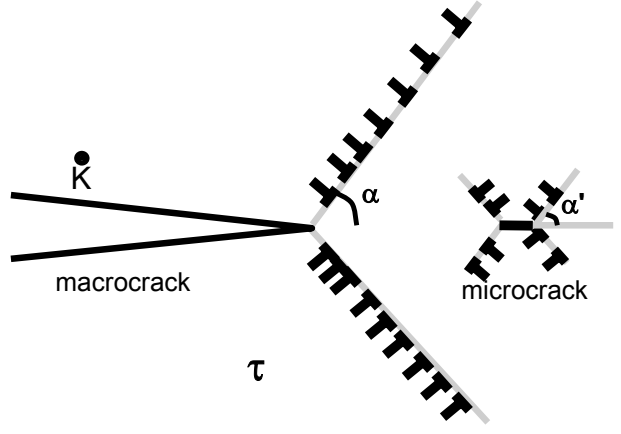

Fig. 1a: The Geometry of the crack and dislocations used in the macrocrack - microcrack simulation model. $\mathrm{K}$ is the applied load at the macrocrack, the slip planes angles are oriented at $\alpha$ and $\alpha$ ' to the crack planes of macrocrack and microcrack respectively.

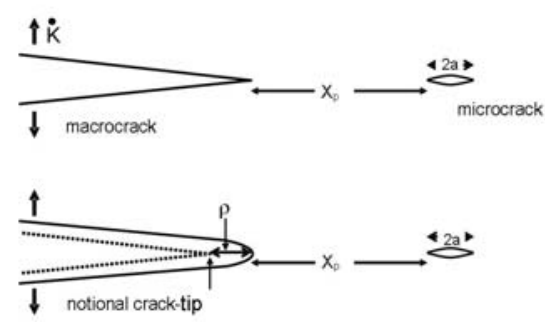

Fig.1b: Schematic illustration of the crack-tip blunting. $\rho$ is the radius of the blunted crack-tip, $2 r$ the microcrack size and $X_{p}$, the distance of the microcrack from macrocrack tip. The distance of the microcrack from the notional sharp crack-tip increases from $X_{p}$ to $X_{p}+\rho$.

increases: (a) the crack tip fields are modified to be that of a blunted crack [12], (b) source position is chosen to be equal to the crack-tip radius, i.e. $x_{0}=\rho$ (c) the notional crack-tip from which the image stress of dislocations is calculated is moved back to the center of curvature of the blunted crack. The arrays of emitted dislocations form the plastic zones of the crack and the crack may also get blunted due to dislocation emission. The plastic zone developed at the macrocrack modifies the field ahead of it to that of an elastic-plastic material with hardening [13]. During loading, dislocations are emitted from source positions when the resolved shear stress reaches a value $2 \tau_{y}$, where $\tau_{y}$ is the friction stress experienced by dislocations and is due to the effect of all type 
of obstacles in dislocation motion including impurities, precipitates, grain boundaries, etc in addition to the lattice friction. The resolved shear stresses are obtained using expressions based on derivations for a finite crack [14] and a semi-infinite crack [15] for respective cases. The emitted dislocations move with a velocity based on the following expression: $\mathrm{v}_{x_{i}}=\left(\frac{\tau_{x_{i}}-\tau_{y}}{\tau_{x_{i}}}\right)\left(\left|\tau_{x_{i}}\right|\right)^{m} \mathrm{~A} e^{(-\mathrm{Ea} / \mathrm{kT})}$ when $\left|\tau_{x_{i}}\right|>\tau_{y}$, and for $\left|\tau_{x_{i}}\right|<\tau_{y}, \quad \mathbf{v}_{x_{i}}=0$. The values for the parameters were obtained by fitting the data of screw dislocation velocities in iron [16]. The value of $m$ has a linear dependence on temperature, $\mathrm{T}, m=400 / T+1.2, A=3.14 \times 10^{-4}$ meters $s^{-1}(M P a)^{-m}$ and $E_{a}=0.316 \mathrm{eV}$. Since the dislocations move only when $\left|\tau_{x_{i}}\right|>\tau_{y}$ they are in their equilibrium positions at any given time, therefore the temperature and strain rate dependence of measured fracture toughness $\left(K_{F}\right)$, plastic zone size $\left(d_{F}\right)$, crack-tip opening displacement, etc. are determined only by the temperature and strain-rate dependence of the friction stress $\left(\tau_{y}\right)$. The applied load is incremented at a rate $d K / d t\left(=0.01 \mathrm{MPa} \sqrt{ } \mathrm{m} \mathrm{s}^{-1}\right)$; a variable time step is used to make sure dislocations move as an array and numerical instabilities are avoided. The distribution of microcrack obtained in the previous section is placed ahead of the macrocrack in the crack plane beyond a distance $X_{\min }=10 \mu m$, (which is about a grain diameter). At each time step, the stress ahead of the crack at distances $X p$ 's (the microcracks are assumed to be at these positions) along the crack plane are calculated. The fracture criterion is the tensile stress $\left(\sigma_{y y}^{p}\right)$ at any of the particle (at distance $X p^{i}$ ) reaching $\sigma_{F}{ }^{i}$ (calculated in the previous stage). The applied load at the macrocrack gives the fracture toughness $\left(K_{F}\right)$.

\section{Results and Discussion}

(a) Microcrack simulation: calculation of microscopic fracture stresses $\left(\sigma_{F}\right)$

A microcrack of configuration shown in the inset of Figure 2 is loaded monotonically, dislocation sources emitting dislocations along four symmetric slip planes $\left(\alpha^{\prime}=70^{\circ} 5^{\prime}\right)$ around the through thickness crack is used. To mimic the triaxial stress field surrounding the microcrack embedded in the plastic zone of a macrocrack, we modified the expressions for the stress fields given in [14] by subtracting the applied normal stress $\left(\sigma_{A y}\right)$ and adding the uniaxial yield stress $\left(\sigma_{y}\right)$ for cases when $\sigma_{A y}>\sigma_{y}$. The dislocations are emitted from source positions. Following an earlier study on the effect of source position [11] here we have chosen the source positions as $4 b,(b=2.54 \AA)$ 


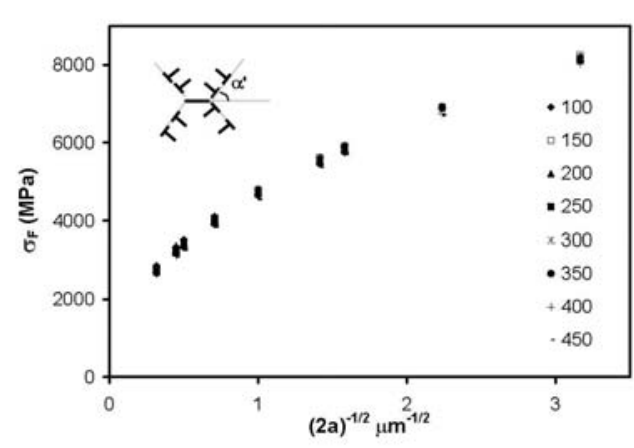

Figure 2a: The microscopic fracture stresses $\left(\sigma_{F}\right)$ as a function of crack size $(a)$, the values of $\sigma_{F}$ is shown for different friction stresses (their values in MPa are shown as labels)

along the slip planes. The stress fields of the emitted dislocations shield the crack-tip from external load. Once moved to their equilibrium position, the amount of shielding from each dislocation is calculated using expressions from [14] and the total shielding at the crack-tip is obtained by summation. The crack-tip stress intensity is calculated at each time step and when it reaches a pre-assigned critical value $k=K_{I c}=1.0 M P a \sqrt{ } m$ (value estimated for $\mathrm{Fe}$ with surface energy of $2 \mathrm{Jm}^{-2}$ ), the crack is assumed to propagate and the corresponding applied load is the microscopic fracture stress $\left(\sigma_{F}\right)$.

The microscopic fracture stress $\left(\sigma_{F}\right)$ is estimated for a range of crack sizes from 0.1 to $5 \mu \mathrm{m}$. Figure 2a shows the $\sigma_{F}$ 's as a function of the inverse square root of crack size $(a)$, it should be noted that the relation is linear for small crack sizes but deviates for large cracks. The $\sigma_{F}$ estimated for different friction stresses is shown in Figure 2b; it should be noted that the fracture stress $\left(\sigma_{F}\right)$ values of any given crack size is practically independent of temperature consistent with many of the experiments (e.g. [7]). From the best fit curve we interpolate the value of $\sigma_{F}$ for a given crack size and friction stress. The value of $\sigma_{F}$ 's thus obtained is used to calculate the macroscopic fracture toughness $\left(K_{F}\right)$.

(b) Macrocrack simulation: Calculation of Fracture Toughness $\left(K_{F}\right)$

Figure 3a shows a typical behavior of the tensile stress at the microcrack $\left(\sigma_{y y}{ }^{p}\right)$ position $X_{P}$ as a function of the applied load $(K)$. The fracture criterion in the simulation is $\sigma_{y y}{ }^{p}$ reaching the critical value $\sigma_{F}$ (calculated in the previous stage) at $X_{P}$. For the case shown here the size of the microcrack is $1 \mu \mathrm{m}$, the rate of loading, $d K / d t=0.01$ $M P a \mathrm{~ms}^{-1}$. As the temperature is increased and yield stress (friction stress) is decreased, the applied stress intensity $K$ required for the tensile stress at $X_{P}$ to reach the critical value $\sigma_{F}$ increases 'exponentially'. Two factors contributing to this rapid 


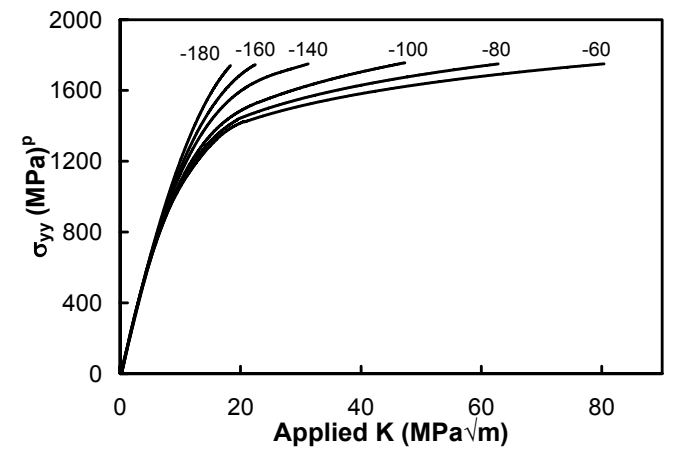

Fig. 3a: The tensile stress at the microcrack $\left(\sigma_{y y}{ }^{p}\right)$ as a function of the $\left(\mathrm{K}_{\mathrm{app}}\right)$ or the simulation time for different yield stresses.

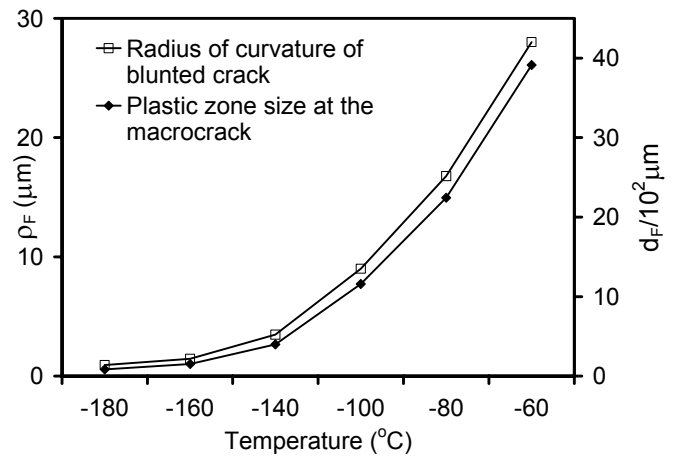

Figure 3b: The crack-tip radius $\left(\rho_{\mathrm{F}}\right)$ and the plastic zone size $\left(\mathrm{d}_{\mathrm{F}}\right)$ calculated at $\mathrm{K}_{\mathrm{F}}$ for each temperature is shown.

increase could be the decrease in the tensile stress at the due to the crack-tip blunting and the increasing effects of stress field (predominantly compressive) from the emitted dislocations. This can be seen in Figure $3 b$ where the plastic zone size $\left(d_{F}\right)$ and the radius of the blunted crack-tip $\left(\rho_{F}\right)$ for each temperature measured at fracture, $K$-applied $=K_{F}$ is shown. The plastic zone size is the distance measured along the slip plane of the

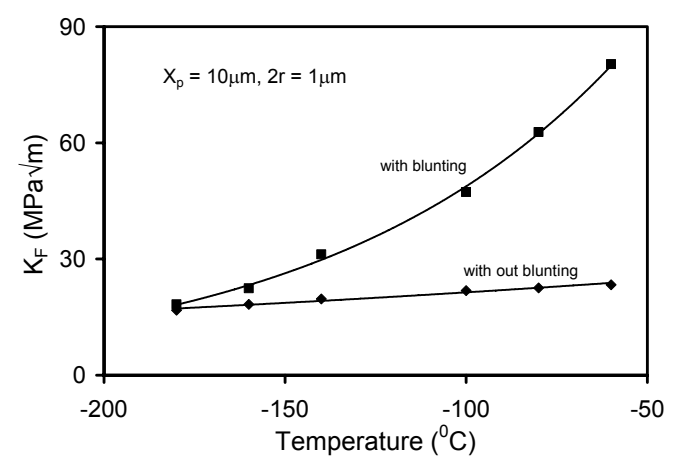

Fig. 4a: The fracture toughness $\left(\mathrm{K}_{\mathrm{F}}\right)$ as a function of temperature for cases with and without blunting: microcrack size $=1 \mu \mathrm{m}$.



Figure 4b: The $J_{2 F}-$ integral calculated for the different temperatures for the blunting case shown in the Figure 5a.

farthest dislocation from the crack-tip. The dislocation source distance $\left(x_{0}\right)$ is chosen as $\rho$ for crack-tip radius greater than $4 b$; else $x_{0}=4 b$. Figure 4 a shows the macroscopic fracture toughness, $K_{F}$, as a function of temperature for cases with and without considering the effects of crack-tip blunting. For the case without blunting the increase in the fracture toughness $\left(K_{F}\right)$ with temperature is small. On the other hand, a sharper increase in the fracture toughness is observed when blunting is accounted for in the simulation. This striking observation emphasizes the significant effect of blunting in the 
increase of fracture toughness with temperature. This exponential increase in the fracture toughness corresponds to the transition from brittle to ductile behavior.

In Figure $4 \mathrm{~b}$ the $J_{2 F}$, the $J_{2}$ - integral value at fracture, calculated from the number of dislocations emitted at the corresponding load $K_{F}$ is shown. $J_{2}$ is defined as the sum of the glide forces on all dislocations around the crack-tip[17]. In this case, the dislocations are in equilibrium against the friction stress $\left(\tau_{y}\right)$ and we can compute $J_{2 F}$ as the product of the total number of dislocations $(N)$ and the friction stress $\left(\tau_{y}\right)$. Considering the fact that $J_{2 F}$ is calculated from the number of dislocations emitted at applied stress intensity factor, $K_{F}$, it is striking to note that the pre-factor of the exponent of the $J_{2 F}$ - temperature curve $(0.0255)$ matches with that of the $K_{F}$-temperature curve (0.0123) in order for the known proportionality between $K^{2}$ and $J$ to hold.

\section{(c) Generation of Microcrack Distribution}

To mimic the Poisson type distribution [11] of microcracks size in the crack plane (mainly originates from the carbides) we generated microcracks with a Poisson distribution of mean radius of $0.5 \mu \mathrm{m}$; and uniformly distributed in a $50 \mu \mathrm{m}$ by $50 \mu \mathrm{m}$ square region. (The $50 \times 50(\mu \mathrm{m})^{2}$ region should correspond to the center of an experimental specimen satisfying the plane strain condition). The density of microcracks is taken to be $0.06 / \mu \mathrm{m}^{2}$, comparable with the microstructures of typical steels. A typical size distribution of the microcracks used is shown in Figure 5b; in the inset the corresponding distribution is shown. The $\sigma_{F}$ 's corresponding to each of these microcracks are then estimated from the interpolation of microscopic fracture stress data shown in Figure 2a, and used as input in the next stage of simulation.

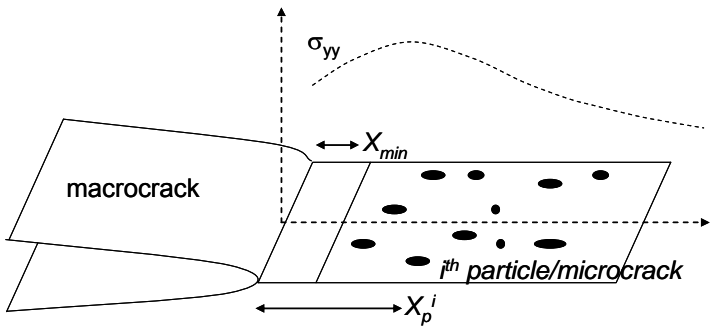

Figure 5a: The Geometry of the model used: A macrocrack with microcracks distributed ahead of it in its crack plane. The propagation of any of the microcracks is the failure criterion in the simulation.

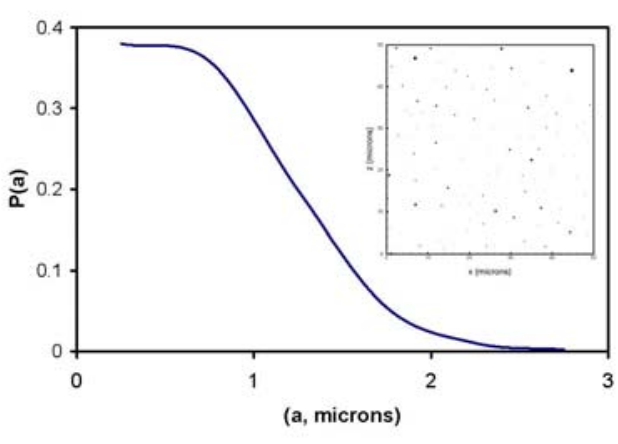

Fig. 5b: The distribution of sizes of the microcracks is shown; a Poisson distribution with mean radius of $0.5 \mu \mathrm{m}$ is used. In the inset a plot of distribution of microcracks uniformly distributed in the crack-plane is shown.

(d) Statistical Analysis of Fracture Toughness $\left(K_{F}\right)$. 
We used six different distributions of microcracks for each friction stress values; here we report the results obtained for friction stress values from 200 to $600 \mathrm{MPa}$. As shown in Figure 4a, when the friction stress is decreased, the applied stress intensity $K$ required for the tensile stress at $X_{P}^{i \text { “s }}$ to reach the critical value $\sigma_{F}^{i}$ increases rapidly.

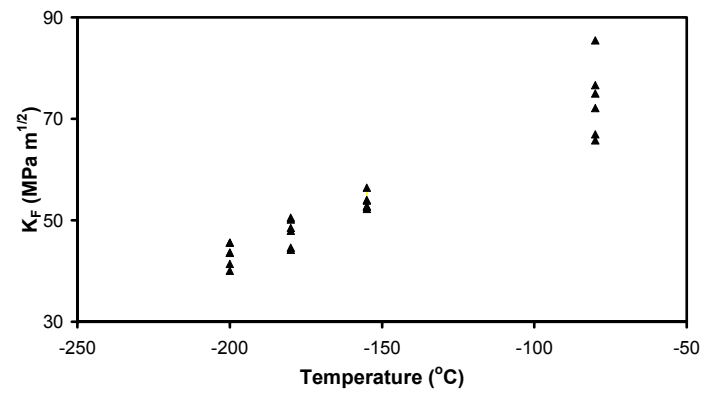

Fig. 6a: The fracture toughness $\left(K_{F}\right)$ as a function of friction stress for cases with six different microcrack configurations is plotted. The mean value curve is also shown.

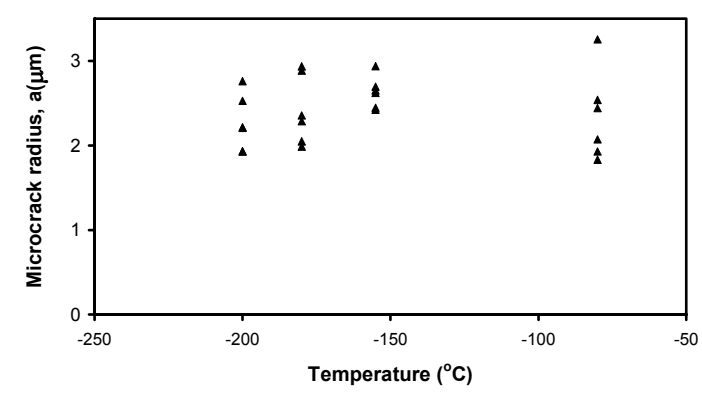

Fig. 6c: The size of microcracks that lead to fracture for different temperatures corresponding to figure 4 a.

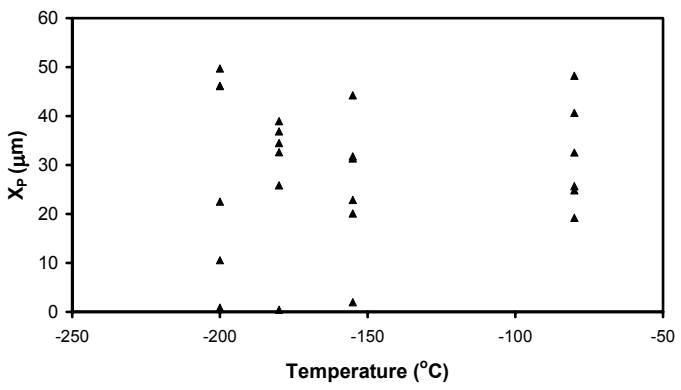

Fig. 6b: The distance of microcracks that leads to fracture in each simulation reported in Figure 4a

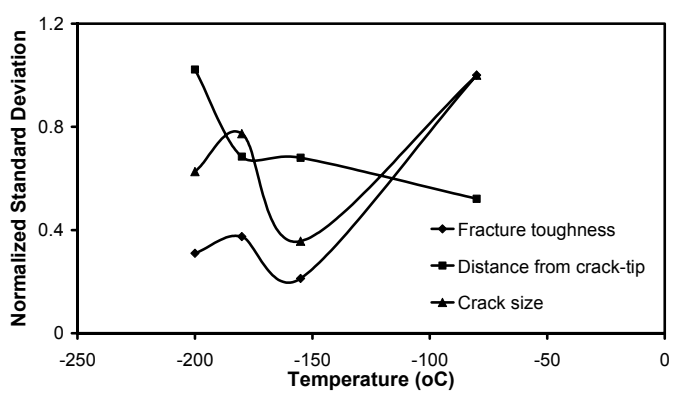

Fig. 6d: The standard deviation of $K_{F}, a$, and $X_{P}$ values respectively plotted in figures $5 \mathrm{a}, 5 \mathrm{~b}$ and $5 \mathrm{c}$.

Two factors contributing to this exponential increase could be the decrease in the tensile stress at the microcrack due to the crack-tip blunting and the increasing effects of stress field (predominantly compressive) from the emitted dislocations. This can be seen in Figure $3 b$, where the number of dislocations emitted $\left(N_{F}\right)$ and plastic zone size $\left(d_{F}\right)$ for each friction stress measured at fracture, $K$-applied $=K_{F}$ is shown. The plastic zone size is the distance measured along the slip plane of the farthest dislocation from the cracktip. This exponential increase in the fracture toughness corresponds to the transition from brittle to ductile behavior.

Figure 6a shows the scatter of the radius of microcracks that leads the fracture. It should be noted that the size of the microcracks that lead to fracture in all the cases are around $2-3 \mu \mathrm{m}$ and the scatter of these values are very narrow; and are independent of the friction stress. On comparing the size of these microcracks with Figure 5b (where a typical size distribution of the crack size is shown), it can be seen that the microcracks 
that lead to fracture are among the largest in any given sample. However it should be noted from Figure 5b, that the distance of these microcracks from the macrocrack-tip varies substantially from case to case and is widely scattered. Even though the $X p$ is widely scattered it is found that it is not correlated with the scatter of measured $K_{F}$ (compare Figures 6a \& 6b); whereas it looks like the crack size distribution is correlated to the $\mathrm{K}_{\mathrm{F}}$ (compare Figures 6a \& 6c) even though their scatter is narrow. Figure 6c shows the normalized (for clarity of display) standard deviation of the values shown in figures $6 \mathrm{a}, 6 \mathrm{~b}$ and $6 \mathrm{c}$ respectively of $K_{F}, X p$ and microcrack size $(a)$. It can be clearly seen that the standard deviation of $K_{F}$ is correlated to microcrack size $(a)$ and are uncorrelated to $X_{p}$.

\section{Summary and Conclusions}

We presented a dislocation simulation model to study the brittle-ductile transition in Ferritic steels. We found two factors that contribute to the sharp increase in the fracture toughness with temperature: (a) the increase in the mobility of the emitted dislocations, and (b) the effect of crack-tip blunting of the marcocrack. However, usually the experimental measurements of fracture toughness with temperature show wide scatter in values. To address this problem of inherent scatter, we carried out a series of Monte Carlo simulation using a distribution of microcracks situated in the crack plane ahead of the macrocrack. On analysing the statistics of these simulations we found that (a) one among the largest microcracks initiates the fracture in each realization, and (b) the inherent scatter arises from the variation in the size of the microcrack that initiates the fracture and not from the variation of its distance from the macrocrack tip.

\section{Acknowledgements:}

This work is supported by US Department of Energy, Office of Fusion Energy, through Grant DE-FG02-03ER54708. SJN would like to thank Prof. Mulugeta Bekele, Addis Ababa University, Ethiopia for useful discussions.

\section{References}

[1] C. J. McMahon Jr, M. Cohen, Acta Metall, 13 (1965) 591.

[2] P. B. Hirsch, S. G. Roberts and J. Samuels, Proc. R Soc. Lond. A, 421 (1989) 25.

[3] P. B. Hirsch and S. G. Roberts, Phil. Mag. A, 64 (1991) 55.

[4] P. B. Hirsch and S. G. Roberts, Phil. Trans. R. Soc. Lond. A 355 (1997) 355.

[5] S. J. Noronha, N. M. Ghoniem, Metall. Mater. Trans. A, 37 (2005) 539.

[6] D. A. Curry and J. F. Knott, Met. Sci., 13 (1979) 341.

[7] P. Bowen, S. G. Druce and J. F. Knott, Acta Metall. 35 (1987) 1735.

[8] S. R. Ortner, C. A. Hippsley, Mater Sci. Tech 12 (1996) 1035.

[9] M. K. Veistinen and V. K. Lindroos, Scripta Metall. 18, (1984) 185. 
[10] P. Bowen and J. F. Knott, Metall. Trans. A, 17 (1986) 231.

[11] K. Wallin, T. Saario and K. Törrönen, Metal Sci. 18 (1984) 13.

[12] M. Creager and P. C. Paris, Int. J. Frac. Mech. 3 (1967) 247.

[13] S. G. Roberts, S. J. Noronha, A. J. Wilkinson and P. B. Hirsch, Acta Mater. 50 (2002) 1229.

[14] S. Wang and S. Lee, Mat. Sci. Engin. A130 (1990) 1.

[15] V. Lakshmanan and J. C. M. Li, Mat. Sci. Engin. A104 (1988) 95.

[16] H. Saka, K. Nada and T. Imura, Crystal Lattice Defects 4, (1973) 45.

[17] M. X. Shi, Y. Huang, H. Gao, Int. J. Plasticity, 20 (2004) 1739. 